\title{
Efficiency, monotonicity and rationality in public goods economies $\star$
}

\author{
M. Ginés and F. Marhuenda \\ Departmento Fundamentos del Análisis Económico, Universidad de Alicante, \\ E-03071 Alicante, SPAIN
}

Received: February 26, 1996; revised version: January 31, 1997

Summary. In economies with public goods, we identify a necessary and sufficient condition for the existence of cost monotonic, Pareto optimal and individually rational mechanisms. These exist if and only if the preferences of the agents satisfy what we call the equal ordering property. We also show that when this condition holds the egalitarian equivalent correspondence is the only cost monotonic selection from the core of the economy. Furthermore, it is unambiguous in the sense that the agents are indifferent among all the allocations in it.

\section{JEL Classification Number: H41.}

\section{Introduction}

One of the central issues of welfare economics is the search for a decision procedure to divide the joint costs associated with a public project. In this paper we adopt the normative approach in that the solution to the cost share problem is determined by some equitable properties which express the sense of fairness of the society.

We shall not review here the rather extensive literature studying the implications of imposing various combinations of axioms on an allocation rule. Notwithstanding, we wish to draw the attention to a remarkably sharp characterization of the egalitarian-equivalent solution (introduced originally by E. A. Pazner and D. Schmeidler [7]) which has been proposed by H. Moulin in [3] for the case of just one public good. This result is in terms of two principles which are very natural in the setting of economies with public

\footnotetext{
${ }^{\star}$ We thank J. Alcalde, S. Chattopadhyay, L. Corchón and an anonymous referee for their comments. Financial support from the Instituto Valenciano de Investigaciones Económicas and from the Ministry of Education project no. PB 94-1504 is gratefully acknowledged. e-mail:paco@merlin.fae.ua.es
}

Correspondence to: F. Marhuenda 
goods. The first one of them is the core concept as introduced by D. Foley in [2]. The Core requirement guarantees that no coalition of agents will raise any objections to the proposed allocation. The second property is "techno logical monotonicity," i.e., if the publicly owned technology gets better, then no agent should suffer a loss. Technological monotonicity was introduced by J. Roemer ([8]) and has been subsequently used to study some solution concepts (see, for example, $[4,5,9])$. In the context of one public and one private good, it has been shown in [3] that the egalitarian equivalent corre spondence is the only selection from the Core of the economy which is cost monotonic. Furthermore, this correspondence is unambiguous because the agents are indifferent among all the egalitarian equivalent allocations.

Due to the interest of this result, it seems very natural to ask whether it can be extended to more general contexts. The purpose of the present work, is to determine when is such an extension possible. Thus, we adopt a setting very similar to the one in [3], except that we allow for the possibility of more than one public good. Unfortunately, we find that with several public goods, there is an obstruction to the compatibility of the axioms we have mentioned above. More precisely, the main result in section 2 shows that there is a mechanism satisfying Pareto efficiency, individual rationality and techno logical monotonicity if and only if the preferences of the agents fulfill what we call the "equal ordering property". This property requires that the agents have the same ordinal preferences when the public goods are consumed for free. On the other hand, when such a mechanism exists then: (i) It must pick an egalitarian equivalent allocation; (ii) the latter form a subset of the core; and (iii) all cost monotonic mechanisms are equivalent, i.e., they provide the same utility profile to the agents. Thus, if (and only if) the equal ordering property holds, one recovers the results in [3] in the sense that the egalitarian equivalent correspondence is not only unambiguous but it is the only cost monotonic selection from the Core of the economy.

The equal ordering condition, which is very natural in the context of one public and one private good (as in [3]), is rather restrictive in general. For example, with quasi linear, strictly increasing preferences in public goods, the equal ordering property is equivalent to the assertion that all the agents have the same ordinal (though not necessarily cardinal) preferences on public goods.

Thus, the main message which stems from the present work is that with several public goods, there are cost monotonic selections from the core only under somewhat stringent restrictions on the preferences of the consumers. Of course, the one public good case is a very relevant one. Hence, the results in [3] are important in that they cover essentially all the cases for which such a selection from the core is possible. Our results also show that the charac terization of the egalitarian equivalent correspondence, in the setting of many public goods, is a question which remains open for future research.

The difference between just one and several public goods is that, in the first case, everybody likes more of the public good. But, with more than one public good to choose from, different agents might differ in their opinions 
about which one should be given priority over the others creating, thus, an additional source of conflict. Under the equal ordering property, these dis crepancies in priority do not arise.

\section{The model}

We follow closely the modeling in [3]. The space of public goods is $X=\mathbb{R}_{+}^{m}$, with $m \geq 1$. These are produced at a cost which is financed by the members of the society. Given two vectors of public goods $x, z \in \mathbb{R}_{+}^{m}, x \geq z$ (resp. $x \gg z$ ) means that $x_{i} \geq z_{i}\left(\right.$ resp. $x_{i}>z_{i}$ ) for every $i=1, \ldots, m$; the notation $x>z$ indicates that $x \geq z$ and $x \neq z$.

We consider economies with one private good and, possibly, more than one public good. The technology available to produce the public goods is described by a cost function $c: X \rightarrow \mathbb{R}_{+}$. The technology is jointly owned by all the members of the society and only one bundle of public goods is eventually produced. We assume that the technology exhibits some bounded returns to scale when producing very large bundles of public goods. Of course, this does not preclude having arbitrarily large increasing returns to scale for public goods within some compact set.

Assumption 2.1 The technology $c: X \rightarrow \mathbb{R}_{+}$is continuous, nondecreasing, satisfies $c(0)=0$ and

$$
\limsup _{\|y\| \rightarrow+\infty} \frac{\|y\|}{c(y)}<+\infty .
$$

Here we use the Euclidean norm $\|y\|=\sqrt{ } \sum_{k 1}^{n} y_{k}^{2}$. For the purposes of computing the limsup we adopt the following convention: Consider the enlarged real line $\mathbb{R}^{*}=\mathbb{R} \cup\{+\infty, \infty\}$ and extend the usual ordering on $\mathbb{R}$ to $\mathbb{R}^{*}$ by defining $\infty<x<+\infty$ for any real number $x \in \mathbb{R}$. We define $\|y\| / c(y)=+\infty$ whenever $c(y)=0$.

We let $N=\{1, \ldots, n\}$ denote the set of agents. Each agent $i \in N$ has preferences over public and private goods represented by a utility function $u_{i}: X \times Y_{i} \rightarrow \mathbb{R}$, where $Y_{i} \subset \mathbb{R}$ is his consumption set of private good. We will write $u_{i}\left(y, t_{i}\right)$ to denote the utility obtained by agent $i \in N$ when the bundle $y$ of public goods is implemented and he has to contribute the amount $t_{i}$ towards its financing. In principle, a negative payment is not excluded, meaning that agent $i \in N$ receives some compensation from other agents for accepting the bundle $y$ instead of another one he might have preferred to it.

Assumption 2.2 For each $i \in N$, the following hold:

(i) The consumption set of private good is a closed interval $Y_{i} \subset \mathbb{R}$ containing zero.

(ii) The utility function $u_{i}: X \times Y_{i} \rightarrow \mathbb{R}$ is continuous non decreasing in the public goods and strictly decreasing in the private good.

(iii) There is a continuous function $\varphi_{i}: X \rightarrow Y_{i}$ such that $u_{i}\left(y, \varphi_{i}(y)\right)$ $=u_{i}(0,0)=0$. The mapping $\varphi_{i}(y)$ verifies 


$$
\limsup _{\|y\| \rightarrow+\infty} \frac{\varphi_{i}(y)}{\|y\|}=0 .
$$

By (i) private transfers of money among the agents might be allowed de pending on whether the consumption set of private goods contains some negative number. By (ii) there are no public bads and (iii) shows that the amount of private good which agents are willing to provide for the con sumption of any fixed bundle of public goods is limited. Note that $u_{i}(y, t)$ is decreasing in the private good to indicate that $t$ denotes a payment. For convenience, we have normalized $u_{i}(0,0)=0$ for each agent $i \in N$. We also see from (ii) and (iii) that, for $i=1, \ldots, n$, the mappings $\varphi_{i}$ are non de creasing and satisfy $\varphi_{i}(0)=0$.

We let $Y=\prod_{i}^{n}{ }_{1} Y_{i}$ and extend the utility functions of the agents to $X \times Y$, by $u_{i}(y ; t)=u_{i}\left(y, t_{i}\right)$, with $(y ; t)=\left(y ; t_{1}, \ldots, t_{n}\right) \in X \times Y$ and $i \in N$. The utility profile of the agents is the mapping $u: X \times Y \rightarrow \mathbb{R}^{n}$ given by $u(y ; t)$ $=\left(u_{1}\left(y, t_{1}\right), \ldots, u_{n}\left(y, t_{n}\right)\right)$.

An economy is a pair $(u, c)$ consisting of an utility profile $u$ and a tech nology $c$. From now on, we fix the set $Y$ and a profile of utilities $u$ satisfying assumptions 2.2. For simplicity, we use the notation $c$ instead of $(u, c)$ to denote an economy. An allocation $(z ; t) \in X \times Y$ is feasible in the economy $c$ for a non empty coalition $S \subset N$ if $c(z) \leq \sum_{i \in S} t_{i}$ for some $t_{i} \in Y_{i}$ with $i \in S$. We simply say that $(z ; t)$ is feasible whenever it is feasible for the grand coalition $N$.

Given a technology $c$ we say that a nonempty coalition $S \subset N$ can im prove upon an allocation $(z ; t)$ if there is another allocation $(y ; s)$, feasible for $S$, such that $u_{i}(y ; s) \geq u_{i}(z ; t)$ for each $i \in S$ with at least some strict in equality. An allocation $(z ; t)$ is individually rational (resp. in Core $(c)$ ) if no agent can improve upon it (resp. if no coalition can improve it). The set of Pareto optimal allocations, $\mathrm{P}(c)$, consists of those feasible allocations which cannot be improved upon by the grand coalition $N$.

Given two vectors $x=\left(x_{1}, \ldots, x_{m}\right), y=\left(y_{1}, \ldots, y_{m}\right)$ in $\mathbb{R}^{m}$, we use the notation $x \vee y=\left(\max \left\{x_{1}, y_{1}\right\}, \ldots, \max \left\{x_{m}, y_{m}\right\}\right)$. We now specify the domain of economies which we will be considering. We want to make sure that there are individually rational allocations in the economies we deal with. For this reason, we let the set $E$ of admissible economies consist of those mappings $c: X \rightarrow \mathbb{R}_{+}$satisfying assumption 2.1 and such that $c(x \vee y) \leq c(x)+c(y)$ for any pair of bundles of public goods $x, y \in X$.

Observe now that for any $c \in E$ the set of individually rational and fea sible allocations in that economy is non empty. Indeed, let $c \in E$ and suppose that for each agent $i=1, \ldots, n$, the vector $\left(x^{i}, t_{i}\right) \in X \times Y_{i}$ is a solution to the problem $\max \left\{u_{i}(z, r):(z, r) \in X \times Y_{i}, c(z)=r\right\}$. (The existence of a solution is guaranteed by Assumptions 2.1 and 2.2.) Let $x=x^{1} \vee x^{2} \vee \cdots \vee x^{n}$. Then $c(x) \leq \sum_{i 1}^{n} c\left(x^{i}\right)=t(N)$. Hence, $(x ; t)$ is feasible for the grand coalition in the economy $c$ and every agent $i \in N$ is least as well off with $(x ; t)$ as with $\left(x^{i}, t_{i}\right)$. 
Definition 2.3 A mechanism is a mapping $R: E \rightarrow X \times Y$ assigning to every technology $c \in E$ an allocation, $R(c)$, feasible in the economy $c$.

A mechanism $R$ is Pareto efficient if $R(c) \in \mathrm{P}(c)$ for every $c \in E$. It is individually rational if $R(c)$ is an individually rational allocation in the economy $c$. The mechanism $R$ is said to be cost monotonic if, given two cost functions $c_{1}, c_{2} \in E$ such that $c_{1}(y) \leq c_{2}(y)$ for every $y \in X$, it assigns allo cations $R\left(c_{j}\right)$, for $j=1,2$, such that $u\left(R\left(c_{1}\right)\right) \geq u\left(R\left(c_{2}\right)\right)$.

We address now the main issue: given a fixed set of agents, are the axioms of cost monotonicity, Pareto efficiency and individual rationality compatible? We need to introduce two additional concepts before we can answer this question completely. The first one of them is the equal ordering property.

Definition 2.4 We say that the agents order the bundles of public goods equally (or that the profile of utilities $u$ satisfies the equal ordering property) whenever for each bundle of public goods $y, z \in X$, if $u_{i}(y, 0)>u_{i}(z, 0)$ for some agent $i \in N$, then $u_{j}(y, 0) \geq u_{j}(z, 0)$ for every other agent $j \in N$.

In other words, the equal ordering property is fulfilled whenever given $y, z \in X$, either $u(y, 0) \geq u(z, 0)$ or $u(z, 0) \geq u(y, 0)$. Thus, if a consumer prefers the bundle of public goods $z$ to the bundle $y$, when both are free, then so do all the other agents. Thus, agents rank the bundles of free public goods in the same way. This property holds trivially in the case of one public good.

Example 2.5 We illustrate this notion in the case of quasilinear utility functions. The utility of each agent $i \in N$ is given by $u_{i}(y, t)=b_{i}(y) \quad t$, where $b_{i}: X \rightarrow \mathbb{R}$ and $b_{i}(y)$ is the utility obtained by agent $i \in N$ whenever he enjoys the bundle of public goods $y \in X$ for free. The equal ordering prop erty is equivalent to the following statement: for each pair of bundles of public goods $y, z \in X$, either $b_{i}(y) \geq b_{i}(z)$ for every agent $i \in N$ or $b_{i}(z) \geq b_{i}(y)$ for every agent $i \in N$.

Secondly, we introduce the concept of egalitarian equivalence. It was originally proposed by E. A. Pazner and D. Schmeidler ([7]) and has been subsequently characterized in [1] and [3].

Definition 2.6 The set of egalitarian equivalent allocations is defined to be

$$
\mathrm{EE}(c)=\{(x ; t) \in \mathrm{P}(c) \text { : there is } z \in X \text { with } u(x ; t)=u(z ; 0)\} \text {. }
$$

The bundle of public goods $z$ appearing in the definition of $\operatorname{EE}(c)$ is the reference bundle for the allocation $(x ; t)$. The set of egalitarian levels, $\operatorname{EL}(c)$ consists of those allocations $z \in X$ for which there is $(x ; t) \in \mathrm{EE}(c)$ such that $u(z ; 0)=u(x ; t)$. A mapping $R: E \rightarrow X \times Y$ is an egalitarian equivalent mechanism if $R(c) \in \mathrm{EE}(c)$ for every $c \in E$.

In the context of one public and one private good, H. Moulin ([3]) has proved that the egalitarian equivalent mechanism: (i) is the only one which is cost monotonic, individually rational and Pareto efficient; (ii) it is unam biguous in the sense that all the agents are indifferent among those alloca tions; and (iii) is a selection from the Core of the economy. It follows that the 
egalitarian equivalent allocations constitute the only cost monotonic selec tion from the Core. The result stated below makes precise the conditions under which those results extend to the case of many public goods.

Theorem 2.7 Let $N=\{1, \ldots, n\}$ be a set of agents whose profile of utilities, $u=\left(u_{1}, \ldots, u_{n}\right)$, satisfies assumption 2.2. Then,

(a) There is a cost monotonic, Pareto efficient and individually rational mechanism $R: E \rightarrow X \times Y$ if and only if $u$ verifies the equal ordering property.

(b) If the equal ordering property holds then $E E(c) \subset \operatorname{Core}(c)$ for every technology $c \in E$ (i.e. any egalitarian equivalent mechanism is a cost monotonic selection from the Core). Furthermore, If $R: E \rightarrow X \times Y$ is any cost monotonic, Pareto efficient and individually rational mechanism then for every technology $c \in E$,

(i) $R(c) \in \mathrm{EE}(c)$.

(ii) The map $u(\cdot, 0)$ is constant on $\operatorname{EL}(c)$. In fact, $u(z, 0)=u(R(c))$ for any $z \in \operatorname{EL}(c)$.

As a consequence, such a mechanism exists only if the agents have exactly the same ordinal preferences when the bundles of public goods are free. This condition, holds clearly for economies with one public good. However, with several types of public goods it limits the existence of individually rational and cost monotonic selections from the set of Pareto optimal allocations.

The second part makes explicit that, whenever the equal ordering prop erty holds, we are back in the setting of [3]. That is, the egalitarian equivalent correspondence is the only cost monotonic selection from the Core. In ad dition the egalitarian equivalent rule is essentially unique in the sense that all the agents are indifferent among the different allocations in it.

The following example underlines the difficulties associated with the egalitarian equivalent allocations when the number of public goods is greater than one. Firstly, in many cases there is a continuum of egalitarian equiva lent allocations which are not individually rational. Secondly, the ones which are individually rational, also form a continuum of allocations yielding dif ferent, non comparable, utility profiles to the agents.

Example 2.8 The economy $c$ consists of two public goods (so $X=\mathbb{R}_{+}^{2}$ ) and two consumers with quasi linear preferences in money given by the utility functions $u_{1}(y ; t)=2 \sqrt{ } y_{1}+2 \sqrt{ } y_{2} \quad t$ and $u_{2}(y ; t)=2 \sqrt{ } y_{1} \quad t$, where $y=$ $\left(y_{1}, y_{2}\right) \in X$. The cost of producing the bundle $y \in X$ of public goods is $c(y)=y_{1}+y_{2}$. It is easy to compute (see [3]) that the set of egalitarian levels is $\operatorname{EL}(c)=\left\{y \in X: 4 \sqrt{ } y_{1}+2 \sqrt{ } y_{2}=5\right\}$.

Only a strict subset of the egalitarian equivalent allocations are individ ually rational. The set of utilities given by individually rational egalitarian equivalent allocations is $U=\left\{\left(v_{1}, v_{2}\right): v_{1}+v_{2}=5, v_{1} \geq v_{2}, v_{1} \geq 2, v_{2} \geq 1\right\}$. Hence, not all the egalitarian levels provide individually rational allocations 
and, furthermore, there are several, non comparable distributions of utilities in the set $U$.

We finish this section with some remarks. There is a related literature, in the context of monotonicity with respect to changes in resources ([6], [10]). The conclusion therein is that Pareto optimality and resource monotonicity are incompatible with other normative properties such as individual rationality from equal division or envy free. The egalitarian equivalent solution has also been characterized by Pareto efficiency, monotonicity and a certain notion of fairness with respect to some commodity in [1]. These authors also show that the equity axiom cannot be imposed on more than one commodity; thus, their results show the strength of the monotonicity axiom in another setting.

We also mention that one could extend the domain of the mechanism to allow for changes in the number of agents and consider the axiom of pop ulation monotonicity. Roughly speaking, when the number of agents in creases, the cost of financing the optimal bundle of goods is shared among more agents. Thus, population monotonicity requires that by increasing the number of agents everybody should be no worse off than before. It is easy to argue that the axioms of population monotonicity and Pareto optimality imply the Core property and, hence, individual rationality. Therefore, The orem 2.7 also holds when we replace "individual rationality" with "popu lation monotonicity."

Finally, under assumptions 2.2 any monotonic selection from the egali tarian equivalent correspondence defines a cost monotonic, Pareto efficient (but of course, not individually rational) mechanism. Thus, the axiom of cost monotonicity alone cannot discriminate among the different egalitarian equivalent allocations.

\section{Proof of Theorem 2.7}

We use the notation $x(S)=\sum_{i \in S} x_{i}$ for a non empty subset $S \subseteq N$ and a vector $x \in \mathbb{R}^{Q}$, with $S \subseteq Q \subseteq N$. The proof of Theorem 2.7 hinges on the following three preliminary lemmas.

Lemma 3.1 Let $i \in N$ and $y \in X$. Then, there is a continuous, non decreasing function $d_{i}: \mathbb{R}_{+} \rightarrow \mathbb{R}_{+}$such that $d_{i}(\lambda)=0$ for $\lambda \leq 1$ and $u_{i}\left(\lambda y, d_{i}(\lambda)\right)=u_{i}(y, 0)$ for $\lambda \geq 1$.

Proof Fix $\lambda \geq 1$ and let $h: \mathbb{R}_{+} \rightarrow \mathbb{R}$ be defined by $h(s)=u_{i}\left(\lambda y, \varphi_{i}(s y)\right)$. Then, $h(\lambda)=u_{i}\left(\lambda y, \varphi_{i}(\lambda y)\right)=u_{i}(0,0) \leq u_{i}(y, 0)$ and, since $\varphi_{i}(0)=0$, we also have that $h(0)=u_{i}(\lambda y, 0) \geq u_{i}(y, 0)$. Since $h$ is continuous, there is $s(\lambda) \in[0, \lambda]$ such that $h(s(\lambda))=u_{i}\left(\lambda y, \varphi_{i}(s(\lambda) y)\right)=u_{i}(y, 0)$. We define $d_{i}(\lambda)=\varphi_{i}(s(\lambda) y)$. Note that $d_{i}(\lambda)$ is uniquely defined because $u$ is strictly increasing in the second argument. Also, $0=d_{i}(1) \leq d_{i}(\lambda) \leq \varphi_{i}(\lambda y)$, since $\varphi_{i}$ is increasing.

In addition, $d_{i}$ is continuous and non decreasing for $\lambda \geq 1$ because it satisfies the equation $u_{i}(y, 0)=u_{i}\left(\lambda y, d_{i}(\lambda)\right)$. Thus, we can extend $d_{i}(\lambda)$ con tinuously to $[0,1]$ by requiring that it vanishes in that interval. 
Lemma 3.2 Let $y \in \mathbb{R}_{++}^{m}$ be a bundle of public goods in the interior of $X$. Then, a technology $c_{y} \in E$ exists such that $u(z ; t)=u(y ; 0)$ for any $(z ; t) \in X \times Y$ which is an individually rational and Pareto efficient allocation in the economy $c_{y}$.

Proof Fix $y \in \mathbb{R}_{++}^{m}$ and for each $i \in N$ choose $d_{i}$ as in Lemma 3.1 and let $d=\max \left\{d_{1}, \ldots, d_{n}\right\}$. Given $x \in X$, let $\lambda(x)=\inf \left\{\lambda \in \mathbb{R}_{+}: x \leq \lambda y\right\}$ and define $c_{y}(x)=2 n \max \{d(\lambda(x)), \lambda(x) \quad 1\}$. To simplify the notation we will drop the subindex and write $c$ to denote $c_{y}$ in the proof of the present Lemma. Note that $c(x)=0$ if $x \leq y$ so $(y ; 0, \ldots, 0)$ is feasible in $c$. In addition, $c(x)>0$ if $\lambda(x)>1$.

We first verify that $c \in E$. Note that $\|x\| \leq \lambda(x)\|y\|$ and $c(x) \geq$ $(\lambda(x)$ 1) for all $x \in X$. Therefore,

$$
\limsup _{\|x\| \rightarrow+\infty} \frac{\|x\|}{c(x)}<+\infty
$$

One checks easily that $\lambda(x \vee z)=\max \{\lambda(x), \lambda(z)\}$, so $c(x \vee z)=\max \{c(x)$, $c(z)\} \leq c(x)+c(z)$. Hence, $c \in E$.

Let now $(z ; t)=\left(z ; t_{1}, \ldots, t_{n}\right) \in \mathrm{P}(c)$ be an allocation which is individually rational. Denote by $t_{i_{0}}=\max \left\{t_{1}, \ldots, t_{n}\right\}$. Since, $0 \leq c(z) \leq t(N)$ we have that

$$
t_{i_{0}} \geq \frac{c(z)}{n} \geq 2 d(\lambda(z)) \geq 0
$$

and it follows that, $t_{i_{0}} \geq 2 d(\lambda(z)) \geq 2 d_{i_{0}}(\lambda(z))$.

First, consider the case $\lambda(z)>1$. If $d_{i_{0}}(\lambda(z))>0$, then $t_{i_{0}}>d_{i_{0}}(\lambda(z))$ so by Lemma 3.1 we have that $u_{i_{0}}\left(z, t_{i_{0}}\right)<u_{i_{0}}\left(z, d_{i_{0}}(\lambda(z))\right) \leq u_{i_{0}}\left(\lambda(z) y, d_{i_{0}}(\lambda(z))\right)$ $=u_{i_{0}}(y, 0)$. Therefore, $(z ; t)$ cannot be individually rational because agent $i_{0}$ would be strictly better off by deviating to the allocation $(y ; 0)$ which is feasible for him.

Hence, we must have that $d_{i_{0}}(\lambda(z))=0$. But then $u_{i_{0}}(y, 0)=u_{i_{0}}(\lambda(z) y, 0)$ $\geq u_{i_{0}}(z, 0) \geq u_{i_{0}}\left(z, t_{i_{0}}\right)$. By individual rationality, $u_{i_{0}}(y, 0)=u_{i_{0}}\left(z, t_{i_{0}}\right)$ $=u_{i_{0}}(z, 0)$. Hence, $t_{i_{0}}=0$ and it follows that $c(z)=0$. But this contradicts that $\lambda(z)>1$.

Therefore, it must be the case that $\lambda(z) \leq 1$ and $z \leq y$. Since $t_{i_{0}} \geq 0$ we have that $u_{i_{0}}\left(z ; t_{i_{0}}\right) \leq u_{i_{0}}(z ; 0) \leq u_{i_{0}}(y, 0)$. But $(z ; t)$ is individually rational and, hence, $u_{i_{0}}\left(z ; t_{i_{0}}\right)=u_{i_{0}}(z ; 0)=u_{i_{0}}(y, 0)$. We conclude that $t_{i_{0}}=0$, so $t=0$ and then $u(z ; t) \leq u(y ; 0)$. Hence, by Pareto optimality, $u(z ; t)=u(y ; 0)$.

It is easy to check that $c_{1} \vee c_{2} \in E$ for any $c_{1}, c_{2} \in E$, where $\left(c_{1} \vee c_{2}\right)(y)=\max \left\{c_{1}(y), c_{2}(y)\right\}$. Using this remark, one can verify that the same proof that is used in [3], also applies here to obtain the following result.

Lemma 3.3 Let $R$ be a Pareto optimal and cost monotonic mechanism. Then for each $c_{1}, c_{2} \in E$ we have that either $u\left(R\left(c_{1}\right)\right) \geq u\left(R\left(c_{2}\right)\right)$ or $u\left(R\left(c_{1}\right)\right) \leq u\left(R\left(c_{2}\right)\right)$.

Now we address the proof of Theorem 2.7. The next Proposition deals with the "only if" implication in part (a) and with (i) and (ii) of part (b) in that Theorem. 
Proposition 3.4 Let $R$ be a cost monotonic, Pareto efficient and individually rational mechanism. Then,

(i) For any economy $c \in E, R(c) \in \mathrm{EE}(c)$.

(ii) For every technology $c$ and every egalitarian level $z \in \operatorname{EL}(c)$ we have that $u(z, 0)=u(R(c))$, i.e. the map $u(\cdot, 0)$ is constant on $\operatorname{EL}(c)$.

(iii) The utility profile $u=\left(u_{1}, \ldots, u_{n}\right)$ satisfies the equal ordering property.

Proof (i) Choose a technology $c \in E$ and let $R(c)=(x ; t)$. Fix $z \in \mathbb{R}_{++}^{m}$ a bundle of public goods which is strictly positive. We will prove that $u(R(c))=u\left(\lambda_{0} z ; 0\right)$ for some $\lambda_{0} \in \mathbb{R}_{+}$.

For each $\lambda \in \mathbb{R}_{+}$we may apply Lemma 3.2 with $y=\lambda z$ to construct $c_{\lambda z} \in E_{1}$ such that $u\left(R\left(c_{\lambda z}\right)\right)=u(\lambda z ; 0)$. By Lemma 3.3,

$$
\text { either } u(R(c)) \geq u(\lambda z ; 0) \text { or } u(R(c)) \leq u(\lambda z ; 0) \text {. }
$$

Let $\lambda$ be large enough so that $\lambda z \geq x$. Since $0 \leq c(x) \leq \sum_{i}^{n} t_{i}$, there must be some agent, say $i_{0} \in N$, such that $t_{i_{0}} \geq 0$. Therefore, $u_{i_{0}}(\lambda z, 0) \geq u_{i_{0}}\left(x, t_{i_{0}}\right)$ and hence $u(\lambda z ; 0) \geq u(R(c))$. Observe also that, by individual rationality, $u(0 ; 0) \leq u(R(c))$. Let $\lambda_{0}=\inf \{\lambda: u(R(c)) \leq u(\lambda z, 0)\} \geq 0$. By continuity, $u_{i}(R(c)) \leq u_{i}\left(\lambda_{0} z, 0\right)$, for each $i=1, \ldots, n$. Suppose some inequality is strict, say $u_{1}(R(c))<u_{1}\left(\lambda_{0} z, 0\right)$. Again, by continuity, there is $\lambda^{\prime}<\lambda_{0}$ close enough to $\lambda_{0}$ such that we still have $u_{1}(R(c))<u_{1}\left(\lambda^{\prime} z, 0\right)$. On the other hand, by the definition of infimum, there must be some other index, say $i=2$, such that $u_{2}(R(c))>u_{2}\left(\lambda^{\prime} z, 0\right)$. But this contradicts equation 3.1. Therefore, $u(R(c))=u\left(\lambda_{0} z, 0\right)$ so $R(c) \in \mathrm{EE}(c)$ and (i) follows. Note that we have also shown that $\operatorname{EL}(c)$ has a non-empty intersection with every half-line of the form $\left\{\lambda z: \lambda \in \mathbb{R}_{+}\right\}$with $z \gg 0$.

(ii) Let now $z \in \operatorname{EL}(c)$ be a strictly positive bundle of public goods. As the argument of the proof of part (i) above shows, one can find $\lambda_{0} \in \mathbb{R}_{+}$such that $u(R(c))=u\left(\lambda_{0} z ; 0\right)$. Since $z \in \operatorname{EL}(c)$ and $R(c)$ is feasible, then $u(z ; 0) \geq u(R(c))$. But $R(c)$ is Pareto optimal, so $u(z ; 0)=u(R(c))$. This proves (ii) for bundles of public goods in $\operatorname{EL}(c) \cap \mathbb{R}_{++}^{m}$. A simple continuity arguments can be used to extend the result to all bundles of public goods in $\operatorname{EL}(c)$.

(iii) Let $i \in N$ be an agent, and let $y, z \in \mathbb{R}_{++}^{m}$ be two bundles of public goods. Suppose that $u_{i}(y, 0)>u_{i}(z, 0)$. Using Lemma 3.2 we construct $c_{y}, c_{z}$ such that $u\left(R\left(c_{y}\right)\right)=u(y ; 0)$ and $u\left(R\left(c_{z}\right)\right)=u(z ; 0)$. By Lemma 3.3, either $u\left(R\left(c_{y}\right)\right) \geq u\left(R\left(c_{z}\right)\right) \quad$ or $\quad u\left(R\left(c_{y}\right)\right) \leq u\left(R\left(c_{z}\right)\right)$. Therefore, $u(y ; 0) \geq u(z ; 0)$. Hence, the equal ordering property holds for $y, z \in \mathbb{R}_{++}^{m}$. By continuity this property also holds for bundles of public goods $y, z \in X$.

To finish we prove the remaining assertions in Theorem 2.7. The equal ordering property is a sufficient condition for the existence of cost monotonic mechanisms. This property by itself also guarantees that the egalitarian equivalent allocations are in the Core of the economy. 
Proposition 3.5 Suppose the equal ordering property holds. Then,

(i) Any egalitarian equivalent mechanism is cost monotonic.

(ii) $E E(c) \subset \operatorname{Core}(c)$.

Proof (i) Let $R$ be an egalitarian equivalent mechanism and let $c_{1} \leq c_{2}$ be two technologies in $E$. For each $i=1,2$, let $R\left(c_{i}\right)=\left(y^{i} ; x^{i}\right) \in \operatorname{EE}\left(c_{i}\right)$ with $u\left(z^{i} ; 0\right)=u\left(y^{i} ; x^{i}\right)$ for some $z^{i} \in X$.

By the equal ordering property, either $u\left(R\left(c_{1}\right)\right)=u\left(z^{1} ; 0\right) \geq u\left(z^{2} ; 0\right)$ $=u\left(R\left(c_{2}\right)\right)$ or $u\left(R\left(c_{1}\right)\right) \leq u\left(R\left(c_{2}\right)\right)$. But, $c_{1}\left(y^{2}\right) \leq c_{2}\left(y^{2}\right)$, so the allocation $R\left(c_{2}\right)$ is feasible in the economy $c_{1}$. Since $R\left(c_{1}\right)$ is Pareto optimal in $c_{1}$, we cannot have that $u\left(R\left(c_{1}\right)\right)<u\left(R\left(c_{2}\right)\right)$. Therefore, $u\left(R\left(c_{1}\right)\right) \geq u\left(R\left(c_{2}\right)\right)$.

(ii) Let $x \in \operatorname{EL}(c)$ and $(y ; t) \in \operatorname{EE}(c)$ so that $u(y ; t)=u(x ; 0)$. Suppose that there is a nonempty coalition $S \subset N$ and an allocation $\left(z ; r^{\prime}\right) \in X \times Y^{S}$ such that $c(z)=r^{\prime}(S)$ and $u_{i}\left(z, r_{i}^{\prime}\right) \geq u_{i}(x, 0)$ for $i \in S$ with some inequality being strict. Consider the allocation $(z ; r) \in X \times Y$, where $r_{i}=0$ if $i \in N \backslash S$ and $r_{i}=r_{i}^{\prime}$ if $i \in S$. Then, $c(z)=r(N)$, so $(z ; r)$ is feasible for $N$.

By the equal ordering property, either $u(z ; 0) \geq u(x ; 0)$ or $u(z ; 0) \leq u(x ; 0)$. However, $u(z ; 0) \geq u(x ; 0)$ is not possible, because we would have that $u(z ; r)>u(x ; 0)$ contradicting that the egalitarian equivalent allocations are Pareto optimal.

Hence, we must have that $u(z ; 0) \leq u(x ; 0)$. Then, for each $i \in S$ we have that $u_{i}(z ; 0) \leq u_{i}(x, 0) \leq u_{i}\left(z, r_{i}\right)$, with some inequality strict. Therefore, $r_{i} \leq 0$ for every $i \in S$ and $r_{i}<0$ for some $i \in S$. But this contradicts that $c(z)=r(S) \geq 0$.

\section{References}

[1] Dutta, B., Vohra, R.: A characterization of egalitarian equivalence. Economic Theory 3, 465-479 (1993)

[2] Foley, D.: Lindahl's solution and the core of an economy with public goods. Econometrica 38, 66-72 (1970)

[3] Moulin, H.: Egalitarian-equivalent cost sharing of a public good. Econometrica 55, 963 976 (1987)

[4] Moulin, H.: A core selection for regulating a single-output monopoly. RAND Journal of Economics 18, 397-407 (1987)

[5] Moulin, H., Roemer, J.: Public ownership of the external world and private ownership of self. Journal of Political Economy 97, 347-367 (1989)

[6] Moulin, H., Thomson, W.: Can everyone benefit from growth? Two difficulties. Journal of Mathematical Economics 17, 339-345 (1992)

[7] Pazner, E. A., Schmeidler, D.: Egalitarian equivalent allocations: A new concept of economic equity. Quarterly Journal of Economics 92, 671-687 (1978)

[8] Roemer, J.: The mismarriage of bargaining theory and distributive justice. Ethics 97, 88110 (1986)

[9] Roemer, J., Silvestre, J.: What is public ownership. Working Paper no. 294, Univ. of California at Davis, Dept. of Economics (1987)

[10] Thomson, W.: Monotonic allocation rules in economies with public goods. Mimeo, University of Rochester, NY (1989) 